\title{
Motivación en el aprendizaje del latín: evaluación de una nueva metodología didáctica
}

Manuel Márquez Cruz - Universidad Complutense de Madrid
Ana María Fernández-Pampillón Cesteros - Universidad Complutense de Madrid

0000-0001-9040-169X

0000-0002-6606-0159

Recepción: 22.07.2019 | Aceptado: 22.11.2019

Correspondencia a través de ORCID: Manuel Márquez Cruz

0000-0001-9040-169X

Citar: Márquez Cruz, M y Fernández-Pampillón Cesteros, AM (2019). Motivación en el aprendizaje del latín: evaluación de una nueva metodología didáctica. REIDOCREA, 8, 431-441.

Financiación: este trabajo ha sido financiado por el Proyecto de Innovación "Innova-Docencia" PIE-164 de la Universidad Complutense de Madrid y gracias al apoyo del Colegio Jesús María.

Resumen: La motivación intrínseca es un factor que incide de forma positiva en la correcta evolución del proceso de aprendizaje de una lengua, toda vez que ayuda a fomentar y mantener en el aprendiente el interés por evolucionar en el conocimiento de dicha lengua. En el caso del latín las nuevas metodologías didácticas han de mostrarse acorde a las inquietudes del alumnado a fin de procurar que la motivación por el estudio de esta lengua no decaiga. Objetivo/Hipótesis. Evaluar el impacto que genera desde la perspectiva de la motivación un método inductivo de aprendizaje del latín que desde hace más de tres años los autores llevan poniendo en marcha en el aula. Método. La muestra estaba compuesta por 11 alumnos de ESO (12-16 años) de un colegio de Madrid y se han utilizado dos encuestas adaptadas en el AMBT de Gardner, al inicio y al final de las sesiones, que medían el grado de motivación. Resultados. Se han encontrado resultados positivos, testados a partir de la realización de una encuesta inicial y otra final. Conclusión. Existe una mejora sustancial en la actitud mostrada por los aprendientes hacia el estudio del latín, factor que ayuda a cumplir con los objetivos didácticos.

Palabras clave: Innovación Educativa

Motivation and Latin Learning: Evaluation of a new didactic methodology

\begin{abstract}
Intrinsic motivation is a factor that influences positively on the correct language learning process. It fosters and makes students be interested in learning that language. Regarding Latin, a language with a limited use, new methodologies and approaches need to fulfill students' curiosity. This way, motivation for the study of that language will not diminish. Objectives/hypotheses. Tests the motivation impact of an inductive method to learn Latin that authors have been using for three years at school. Method. The sample was composed of 11 ESO students (12-16 years old) from a school in Madrid and some questionnaires, adapter from Gardner's AMBT, were used at the beginning and end of the sessions in order to measure students' motivation. Results. Have been found positive results, tested at the beginning and at the end of the school year. Conclusion. There is a meaningful improvement concerning students' attitude towards the learning of Latin, a factor that helps to fulfill the learning objectives established.
\end{abstract}

Keywords: Educational Innovations

\section{Introducción}

Durante el proceso de enseñanza de una lengua, el estímulo de la motivación del aprendiente se concibe como uno de los elementos clave a la hora de afrontar con ciertas garantías de éxito el inicio, la continuidad y la culminación dicho proceso didáctico. En el caso del estudio del latín, el hecho de no concebirse como una lengua al uso -con la salvedad de aquellos contextos comunicativos excepcionales que sirven como argumento para negar su calificación de "lengua muerta": la comunicación entre humanistas, foros, revistas, emisoras de radio, grupos de conversación y su consideración como lengua oficial del estado del Vaticano - hacen, si cabe, más necesario recurrir a técnicas y metodologías que favorezcan la motivación del aprendizaje de esta lengua, ya sea desde una perspectiva intrínseca ya extrínseca, con el fin de incidir de manera positiva en los resultados académicos, especialmente 
en el ámbito de las Enseñanzas Medias. Dentro del Proyecto de Innovación Educativa PIE 164:2018_19 de la Universidad Complutense de Madrid, se ha desarrollado una metodología propia para la enseñanza del latín en estadios iniciales que ha contribuido a mejorar el rendimiento académico de los alumnos que cursan la asignatura de latín en $4^{\circ}$ de ESO (Márquez y Chaves, 2016; Márquez y Fernández-Pampillón, 2016), siendo el objetivo del presente estudio, evaluar la influencia de dicha metodología en la mejora de la motivación intrínseca.

Nuestra propuesta metodológica es de carácter inductivo y cognitivo y se basa en la teoría lingüística de valencias (Tesnière, 1959) y en una adaptación de los rasgos semánticos de Lyons (1980) descritos en su descripción de las unidades de primer, segundo y tercer orden (Márquez 2015). La idea básica es partir de los conocimientos semánticos del idioma materno para entender o construir frases en latín. Se utiliza la metáfora del rompecabezas para explicar cómo construir el significado de las oraciones o cómo crear nuevas oraciones. Las oraciones se presentan como rompecabezas en los cuales el verbo es la pieza central de la oración. El verbo se identifica como elemento principal de la oración, en torno al cual se forma una complementación obligatoria (argumentos verbales) y una optativa (satélites) que proporcionan un sentido completo a la frase. Prescindiendo de etiquetas como Sujeto, Complemento Directo, Complemento Indirecto, etc. -que muchas veces el estudiante no tiene claras ni siquiera en su lengua materna -la complementación se aprende a partir del significado del verbo y la morfología de su complementación argumental, dando lugar a un aprendizaje cognitivo de tipo inductivo que se estructura y asienta a través de la práctica y comparación entre unidades verbales que presentan un mismo o similar comportamiento morfológico y semántico.

Esta metodología se ha implementado utilizando dos recursos didácticos: (i) un curso en abierto formado por una serie de lecciones digitales y, (ii) un diccionario didáctico digital de latín también en abierto (Diccionario Didáctico Digital de Latín), cuya estructura lexicográfica innovadora se ajusta al método didáctico.

Con respecto al curso, su estructura y su diseño favorecen no solo el aprendizaje presencial, sino que permite un aprendizaje en modo $b$-learning o autoformativo a distancia. El curso se ha organizado en lecciones autocontenidas. Cada lección se compone de una explicación en tres formatos: video, audio o texto con una duración de en torno a 10 minutos, una actividad de autoevaluación de repaso y unos ejercicios para ahondar en el estudio de la lección. Los ejercicios incluyen prácticas de traducción inversa tradicionales (español-latín) con retroalimentación.

En lo que se refiere al diccionario, las entradas de las unidades léxicas verbales especifican: (i) el o los significados del verbo, (ii) el número de complementos obligatorios (valencia), (iii) su morfología y sus rasgos semánticos según una ontología que responde a su clasificación como entidades humanas, animales, concretas, abstractas o de lugar y (iv) ejemplos que clarifican el comportamiento semántico y morfológico de dichas unidades léxicas. Del resto de lemas, esto es, sustantivos, pronombres, adjetivos, adverbios, preposiciones, conjunciones e interjecciones, se proporciona una información semántica básica para construir o de-construir los "rompecabezas-frase". El diccionario permite, como novedad lexicográfica, una navegación taxonómica por el mismo atendiendo a la información semántica de los lemas, así como las búsquedas tradicionales basadas en la forma de la palabra en latín o español.

Este estudio se ha realizado utilizando como marco teórico el modelo socioeducativo propuesto por Gardner (1985) y probado en diferentes estudios empíricos durante más 
de cuatro décadas (Sayadian y Lashkarian, 2010; Masgoret y Gardner, 2003, entre otros).

Según el modelo de Gardner, la motivación y las actitudes del alumno son dos factores determinantes para lograr buenos resultados de aprendizaje de nuevas lenguas. La motivación, por un lado; existe cuando el alumno está orientado hacia el objetivo de aprender una nueva lengua. Aunque la orientación del alumno es un requisito para la motivación, no garantiza que el alumno logre el objetivo, es decir, que esté suficientemente motivado (Gardner, 1985; Gardner y Tremblay, 1994). Así, Gardner propone que la motivación es una combinación de (Gardner y Tremblay, 1994): (1) esfuerzo, (2) deseo por alcanzar el objetivo de saber la nueva lengua (intensidad del deseo), y, (3) actitud positiva hacia el aprendizaje de la nueva lengua. Gardner, además, propone considerar que la orientación y la motivación del alumno pueden tener dos componentes o constructos: el integrativo y el instrumental. El constructo integrativo se refiere, básicamente, al deseo del aprendiente por adquirir la lengua para comunicarse con el grupo de hablantes. El instrumental se refiere al deseo de ganar reconocimiento social o ventajas económicas a través o mediante el conocimiento de la nueva lengua (Gardner y Lambert, 1972). Aunque la mayor parte de los estudios realizados sobre motivación se han centrado en la motivación integrativa, la motivación es, realmente, una combinación dinámica de ambos componentes, integrativo e instrumental (Gardner y Tremblay, 1994). Finalmente, es importante destacar que la motivación es un atributo interno (o conjunto de atributos internos) del individuo que puede ser influido por fuerzas externas. Esto significa que merece la pena buscar "las fuerzas externas" que pueden mejorar la motivación del aprendiente, y, en consecuencia, facilitar el logro de la adquisición de la nueva lengua.

La actitud, por otro lado, es una causa de la motivación. Se distingue entre actitudes hacia la situación o contexto de aprendizaje y actitudes de integración (Masgoret y Gardner, 2003). Ambas actitudes, según el modelo socioeducativo de Gardner son dos variables correlacionadas que explican o influyen en la motivación para aprender una lengua extranjera. La motivación, por su parte, es un factor responsable del nivel de éxito del aprendizaje. Las relaciones entre orientación, actitudes y motivación han sido objeto de numerosos trabajos, pero ninguno las explica de forma definitiva por la contextualización particular o especificidad de dichos estudios. En todo caso, este trabajo se limita a estudiar si existe un cambio en la motivación o actitudes de los estudiantes hacia el aprendizaje del latín cuando se utiliza la metodología didáctica presentada en la sección de introducción, sin entrar en las posibles relaciones entre ellas.

\section{Objetivos o hipótesis}

El objetivo general de esta evaluación es, por lo tanto, comprobar si el uso de la propuesta metodológica de iniciación al latín tiene un efecto de mejora en la motivación de los alumnos

Teniendo en cuenta el marco teórico de actitud-motivación presentado en la sección anterior, dividimos el objetivo general en los siguientes objetivos concretos:

1. Establecer el nivel promedio de motivación y actitudes del grupo de aprendientes antes y después de la aplicación de la nueva metodología didáctica. 
2. Establecer el promedio general de cada constructo de la motivación y actitudes en cada uno de sus indicadores antes y después de la aplicación de la nueva metodología didáctica.

3. Determinar el peso de cada indicador en cada constructo y de cada constructo en el promedio general de la motivación antes y después de la aplicación de la nueva metodología didáctica.

4. Determinar si se puede detectar un cambio significativo en la motivación o actitudes a nivel global y a nivel de cada constructo e indicador.

La consecución de estos objetivos específicos permitirá responder a las preguntas:

- ¿Cuál es la motivación y actitud inicial del grupo de alumnos para aprender nuevas lenguas?

- ¿Cuál es la motivación y actitud inicial de los alumnos para aprender latín?

- ¿Ha influido el uso de la metodología en la actitud de alumnos acerca de aprender nuevas lenguas?

- ¿Ha influido el uso de la metodología en la motivación de alumnos para de aprender nuevas lenguas?

La hipótesis de trabajo es que el uso de la metodología didáctica de iniciación al latín propuesta tiene un efecto de mejora en la motivación o actitudes de los aprendientes.

\section{Métodos}

\section{Participantes}

La población son los alumnos de educación secundaria de los niveles de $1 \mathrm{er}$. a $4^{\circ}$ de la ESO que nunca han estudiado latín. En España, sólo los alumnos de $4^{\circ}$ de la ESO que eligen la rama de Artes y Humanidades son los que se inician, ese curso, en los estudios del latín. La muestra se toma en el Colegio Jesús María entre un total de, aproximadamente, 340 alumnos de ESO.

El tamaño de la muestra es de 11 alumnos, 5 varones y 6 mujeres. Esta muestra se ha formado con los alumnos que, voluntariamente han escogido la oferta formativa de lengua latina. El nivel de rendimiento académico del grupo es medio (calificaciones finales de curso entre 5-6 puntos sobre 10). La distribución de edades un alumno de 12 años, 3 de 13 años, 5 de 14 años, uno de 15 años y uno de 16 años.

\section{Instrumentos}

Adaptación del cuestionario AMTB -Attitude Motivation and Test Battery- (Gardner, 2004) al latín y al reducido tiempo de la acción formativa. El tiempo disponible para responder al cuestionario era de 15 minutos aproximadamente. Por ello se seleccionaron 29 afirmaciones de las 104 del cuestionario AMTB original: 52 en positivo y 3 en negativo para el control de la consistencia de las respuestas (las preguntas 6,7 y 15). Las afirmaciones seleccionadas cubren los 6 constructos y 11 indicadores, aunque los constructos "Actitud frente a la acción formativa" y "Ansiedad" se han reducido al mínimo porque: (i) son los menos relacionados con el objetivo de la investigación que es la evaluación del método didáctico, y, (ii) el tiempo de la acción formativa es muy reducido con lo que la influencia en la motivación-actitud es esperable que sea mínima. La tabla 2 muestra una síntesis de las modificaciones junto 
con el total de afirmaciones del AMTB (en positivo y negativo) en cada indicador para facilitar la comparativa. La escala Likert se tomó de 0 a 5.

\begin{tabular}{|c|c|c|c|}
\hline Constructo & INDICADORES & $\begin{array}{l}\text { Afirmación } \\
\text { (en positivo o } \\
\text { negativo) }\end{array}$ & $\begin{array}{l}\text { Total de afirmaciones en } \\
\text { test AMTB }\end{array}$ \\
\hline \multirow[t]{3}{*}{ Integrativo } & 1.Interés en idiomas extranjeros & Preguntas $4,6,8,9$ & 10 \\
\hline & $\begin{array}{l}\text { 2. Actitud hacia la comunidad de hablantes } \\
\text { de latín }\end{array}$ & Preguntas 5,22 & 8 \\
\hline & 3. Orientación integrativa hacia el latín & $\begin{array}{l}\text { Preguntas 2, 8, 12, } \\
13,18\end{array}$ & 4 \\
\hline Instrumental & 4. Orientación instrumental del aprendizaje & $\begin{array}{l}\text { Preguntas 11, } \\
14,16,17,20\end{array}$ & 4 \\
\hline \multirow[t]{3}{*}{ Motivacional } & 5. Comportamiento sobre el esfuerzo & Pregunta 1 & 10 \\
\hline & 6. Deseo de aprender latín & $\begin{array}{l}\text { Preguntas } 3,7,10, \\
24\end{array}$ & 10 \\
\hline & 7. Actitud hacia el aprendizaje de latín & Preguntas 15,19, 21 & 10 \\
\hline \multirow{2}{*}{$\begin{array}{l}\text { Actitud hacia la acción } \\
\text { formativa }\end{array}$} & 8. Evaluación del profesor & Pregunta 25 & 10 \\
\hline & 9. Evaluación del curso de latín & Pregunta 23 & 10 \\
\hline \multirow[t]{2}{*}{ Ansiedad frente al latín } & 10. Ansiedad en el uso del latín & Pregunta 26 & 10 \\
\hline & Ansiedad frente a la clase de latín & - & 10 \\
\hline Estimulo de los padres & 11. Estímulo de los padres & Preguntas $27-31$ & 8 \\
\hline
\end{tabular}

También se realiza una encuesta al inicio y final de la aplicación del método didáctico de iniciación al latín.

\section{Diseño del estudio}

Se trata de un estudio experimental de campo e intrasujetos. Se somete a un grupo de alumnos en un contexto real, un curso de verano, a un tratamiento experimental, el método de iniciación al latín. Se observa la motivación y actitud del grupo de forma cualitativa y se plasman las observaciones en notas de campo diarias, después de cada sesión de aprendizaje. Se mide, mediante una test repetido antes y después del curso, basado en AMBT, si este tratamiento ha tenido efectos significativos en las variables motivación y actitud.

\section{Procedimiento}

El experimento se realiza en el marco de un seminario de verano, una vez terminado el curso oficial, celebrado en la segunda semana del mes de junio. El seminario tiene una duración de cinco días seguidos, de martes a viernes y lunes. El seminario constaba de varias ofertas formativas, en materias diversas (lenguas, deporte, ciencias...) cada una organizada en seis sesiones de 45 a 50 minutos. Entre las ofertas formativas se encontraba "Iniciación a la lengua latina". El seminario estaba dirigido a estudiantes de $1^{\circ}, 2^{\circ}, 3^{\circ}$ y $4^{\circ} \mathrm{ESO}$ (entre 12 y 16 años) y los alumnos elegían forma voluntaria las ofertas formativas.

En la oferta formativa de "Iniciación al latín" el modo de enseñanza fue b-learning: en la clase presencial se trabajaba tanto en una plataforma e-learning (Moodle) que contenía las explicaciones en formato de video lección y los ejercicios de autoevaluación, como en el aula presencial resolviendo las dudas y completando los ejercicios con el profesor y los compañeros. La tabla 2 muestra la distribución de las sesiones por días, y un resumen de las actividades didácticas, las observaciones de campo y la actitud de los alumnos. 


\begin{tabular}{|c|c|c|c|c|}
\hline Día & Sesión & Actividades & Observaciones & Actitud \\
\hline 1 & $1^{a}$ & $\begin{array}{l}\text { Presentación, encuesta inicial, actividad } \\
\text { de absorber: lección } 1 \text { (valencias) }\end{array}$ & $\begin{array}{l}\text { La video lección se para en dos ocasiones y se } \\
\text { preguntan dudas y matizan cuestiones técnicas. }\end{array}$ & $\begin{array}{l}\text { Atentos e } \\
\text { interesados }\end{array}$ \\
\hline \multirow[t]{2}{*}{2} & $2^{\mathrm{a}}$ & Actividad de absorber: lección 2 (casos) & $\begin{array}{l}\text { Tercera hora de seminario. La video lección se } \\
\text { detiene en } 3 \text { ocasiones para explicar y contestar } \\
\text { a preguntas. Se explica la caracterización léxica } \\
\text { de los argumentos. }\end{array}$ & $\begin{array}{l}\text { Atentos e } \\
\text { interesados }\end{array}$ \\
\hline & $3^{\mathrm{a}}$ & $\begin{array}{l}\text { Actividad de hacer (cartulinas y por } \\
\text { grupos: dados } 5 \text { verbos, reconstruir la } \\
\text { valencia, dar forma a los argumentos con } \\
\text { sintagmas de la lengua española. } \\
\text { Construir una frase con esos sintagmas y } \\
\text { verbo. Señalar cual sería el caso de los } \\
\text { argumentos si la frase estuviera en latín). } \\
\text { Actividad de absorber: } \\
\text { lección } 3 \text { (1era. Declinación) }\end{array}$ & Cuarta hora de seminario. & $\begin{array}{l}\text { Cansados (2 } \\
\text { horas } \\
\text { seguidas es } \\
\text { inadecuado } \\
\text { para los } \\
\text { alumnos de } \\
1^{0}, 2^{\circ}, 3^{\circ} \text { y } 4^{\circ} \\
\text { ESO). } \\
\text { A pesar de } \\
\text { ello, por lo } \\
\text { general, han } \\
\text { estado } \\
\text { atentos. }\end{array}$ \\
\hline \multirow[t]{2}{*}{3} & $4^{a}$ & Actividad de absorción: cultura romana & $\begin{array}{l}\text { La experiencia de las sesiones anteriores nos } \\
\text { hace tomar la decisión de intercalar sesiones de } \\
\text { cultura con gramática. Por otro lado, la } \\
\text { capacidad de atención de los alumnos ( } 1^{\circ} \text { ESO- } \\
4^{\circ} \text { ESO) se ha visto mermada debido a la } \\
\text { condensación de contenidos en sesiones } \\
\text { anteriores. Se sugiere ampliar en el tiempo cada } \\
\text { una de las sesiones lingüísticas y dedicarle dos } \\
\text { sesiones a cada una de las lecciones. }\end{array}$ & $\begin{array}{l}\text { Atentos e } \\
\text { interesados }\end{array}$ \\
\hline & $5^{a}$ & $\begin{array}{l}\text { Actividad de repaso lección } 3 \text {. Actividad } \\
\text { de hacer: autoevaluación lección } 3 . \\
\text { Actividad de hacer: búsqueda de } \\
\text { palabras en el diccionario } \\
\text { Actividad de hacer: ejercicios de poner en } \\
\text { práctica el uso del diccionario y los } \\
\text { principios lingüísticos aprendidos } \\
\text { (morfología y semántica). } \\
\text { Actividad de absorber: Diccionario } \\
\text { Didáctico Digital de Latín }\end{array}$ & $\begin{array}{l}\text { Durante la ejecución de los ejercicios el profesor } \\
\text { se pasea por las mesas de trabajo para resolver } \\
\text { dudas. Las dudas tienen que ver con errores en } \\
\text { los ejercicios interactivos de la video lección }\end{array}$ & $\begin{array}{l}\text { Atentos y } \\
\text { participativos }\end{array}$ \\
\hline 4 & $6^{a}$ & $\begin{array}{l}\text { Actividad de hacer: continuación de los } \\
\text { ejercicios de poner en práctica el uso del } \\
\text { diccionario y los principios lingüísticos } \\
\text { aprendidos (morfología y semántica) } \\
\text { Actividad de absorber: lección } 4 \text {. (el } \\
\text { verbo) } \\
\text { Actividad de hacer: continuación de } \\
\text { ejercicios para poner en práctica con } \\
\text { diccionario } \\
\text { Los alumnos afirman haber asimilado el } \\
\text { método. Afirman haber captado la } \\
\text { dinámica y comienzan a entender el } \\
\text { funcionamiento de la lengua latina (hay } \\
\text { un alumno que dice que le resulta más } \\
\text { fácil el alemán que el latín). Sugieren que } \\
\text { los ejercicios queden grabados, a fin de } \\
\text { que puedan acceder a los mismos en un } \\
\text { futuro para repasar. }\end{array}$ & $\begin{array}{l}\text { Durante el visionado lección } 3 \text { el profesor } \\
\text { interrumpe para comprobar si se está } \\
\text { comprendiendo } \\
\text { Trabajan por parejas (problemas con los } \\
\text { ordenadores. La sesión se dilata y al final tienen } \\
\text { que trabajar por parejas). } \\
\text { Se tiene que interrumpir la realización de los } \\
\text { ejercicios en línea por la lentitud de la conexión. } \\
\text { Los alumnos afirman haber asimilado el método. } \\
\text { Afirman haber captado la dinámica y comienzan } \\
\text { a entender el funcionamiento de la lengua latina } \\
\text { (hay un alumno que dice que le resulta más fácil } \\
\text { el alemán que el latín). Sugieren que los } \\
\text { ejercicios queden grabados, a fin de que puedan } \\
\text { acceder a los mismos en un futuro para repasar. }\end{array}$ & $\begin{array}{l}\text { Atentos y } \\
\text { participativos }\end{array}$ \\
\hline 5 & - & Realización de la encuesta final & & \\
\hline
\end{tabular}




\section{Análisis de datos}

Estudio Descriptivo.

\section{Resultados}

En la tabla 3 se muestra los valores iniciales y finales promedio por cada indicador y constructo. La desviación estándar y la desviación media del total es de 0,55 en los valores totales finales. La varianza 0,33.

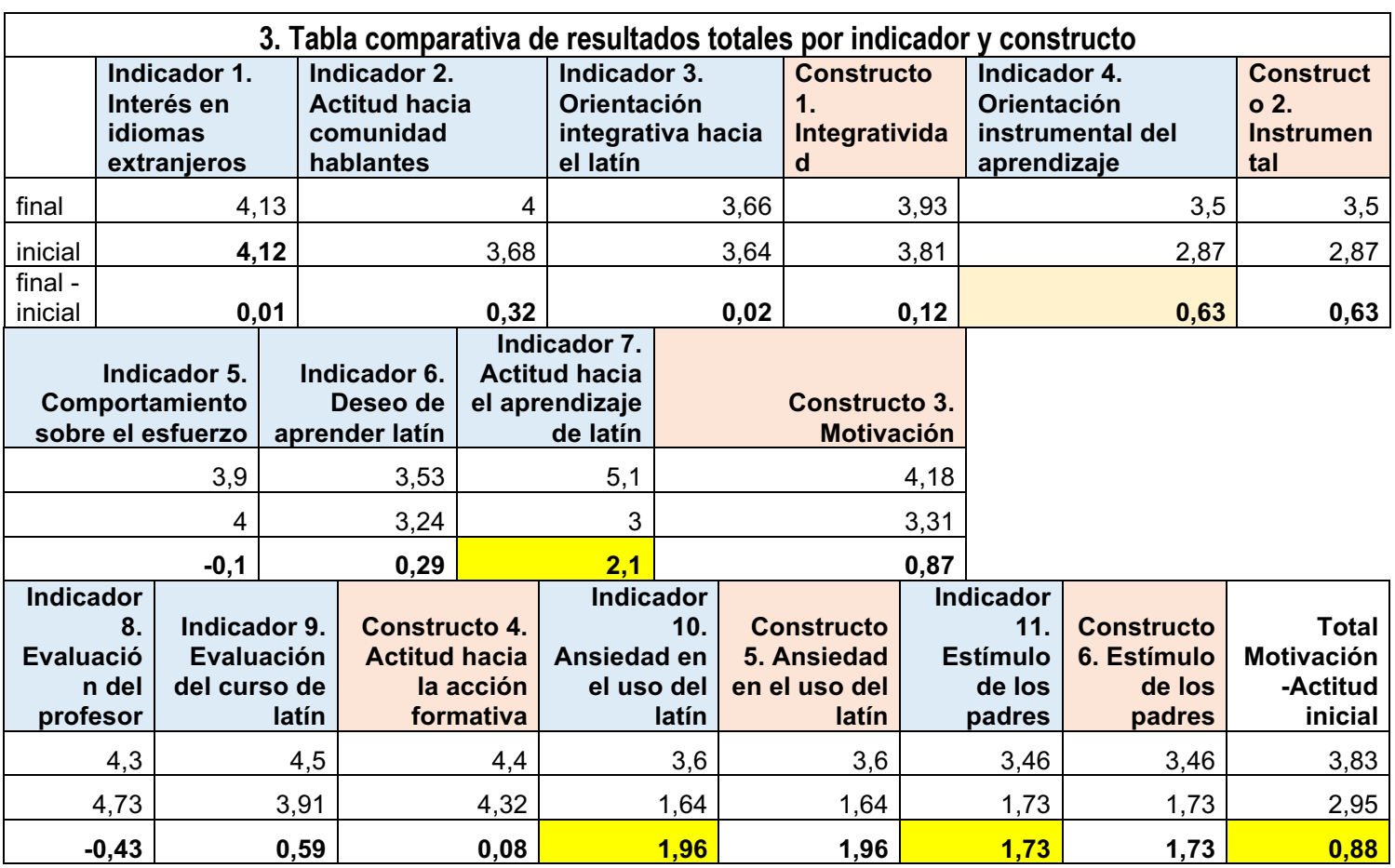

En la figura 1, se muestra el nivel promedio de motivación y actitud del grupo de aprendientes antes y después del tratamiento experimental (objetivo específico 1)

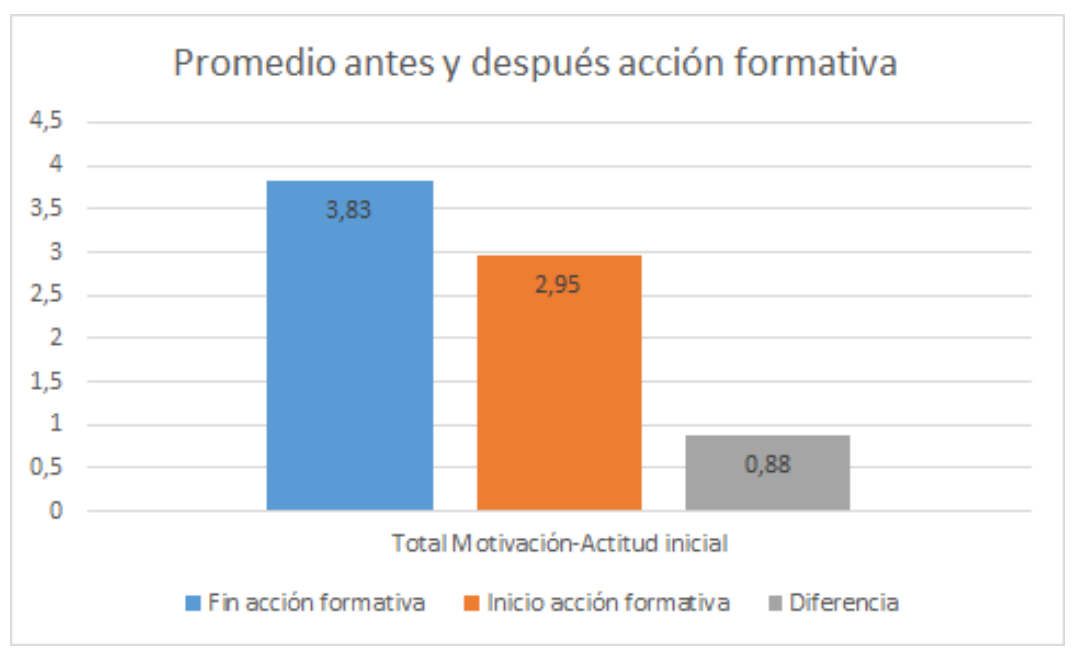

Figura 1. Comparativa de la motivación-actitud antes y después de la acción formativa

En la figura 2 se expone el promedio general de cada constructo en cada uno de sus indicadores antes y después del tratamiento experimental (objetivo específico 2). 

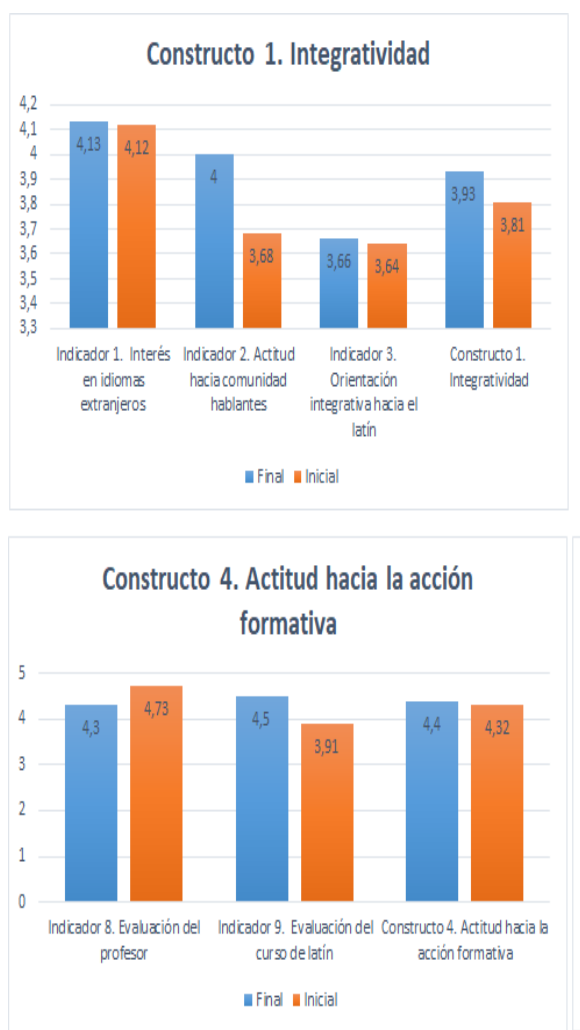

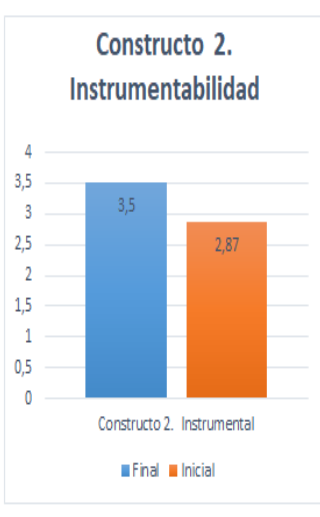

Constructo 5. Ansiedad en el uso del latín

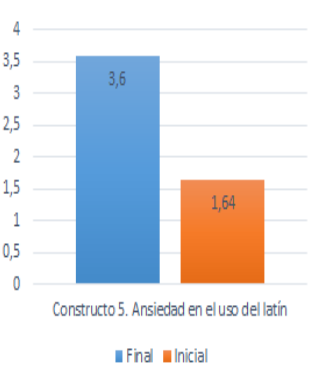

Constructo 3. Motivación

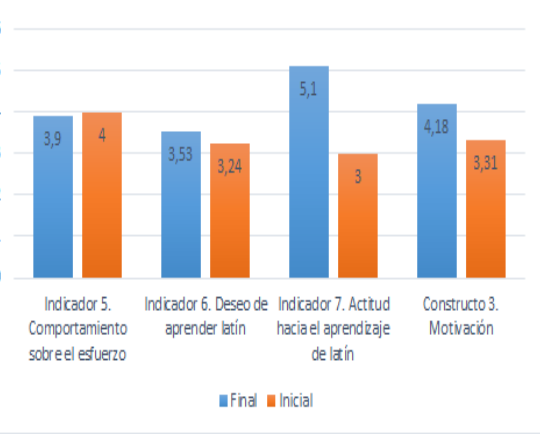

Constructo 6. Estímulo de los padres

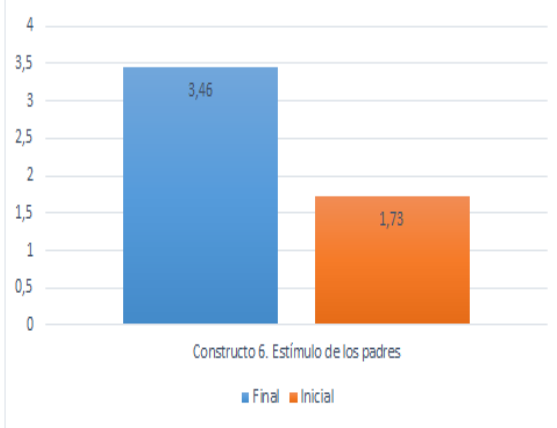

Figura 2. Comparativa de los valores de los indicadores y constructos antes y después de la acción formativa

En las figuras 3 y 4 , se observan el peso de cada indicador en cada constructo y de cada constructo en el promedio general de la motivación antes y después del tratamiento experimental.
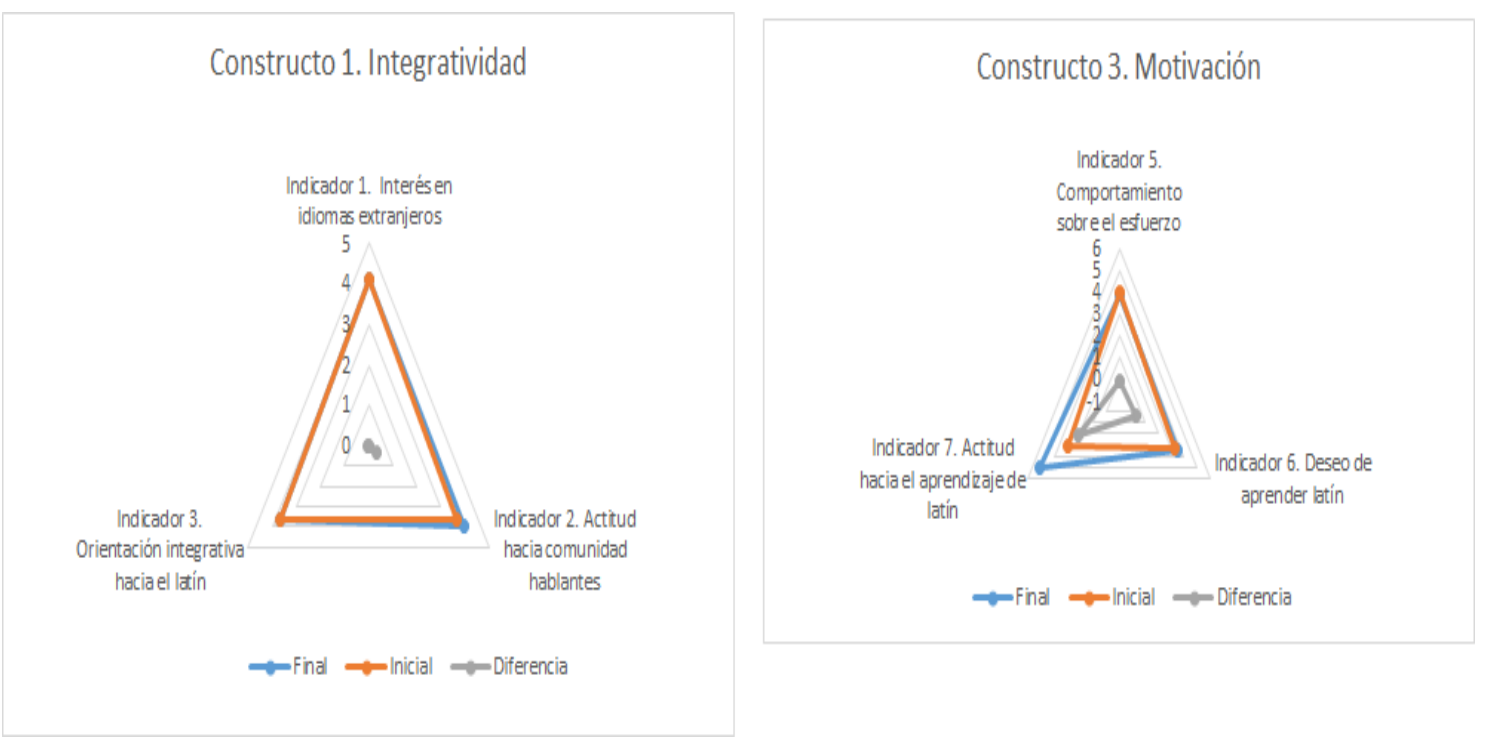

Figura 3. Comparativa de pesos de los indicadores en los constructos con varios indicadores 


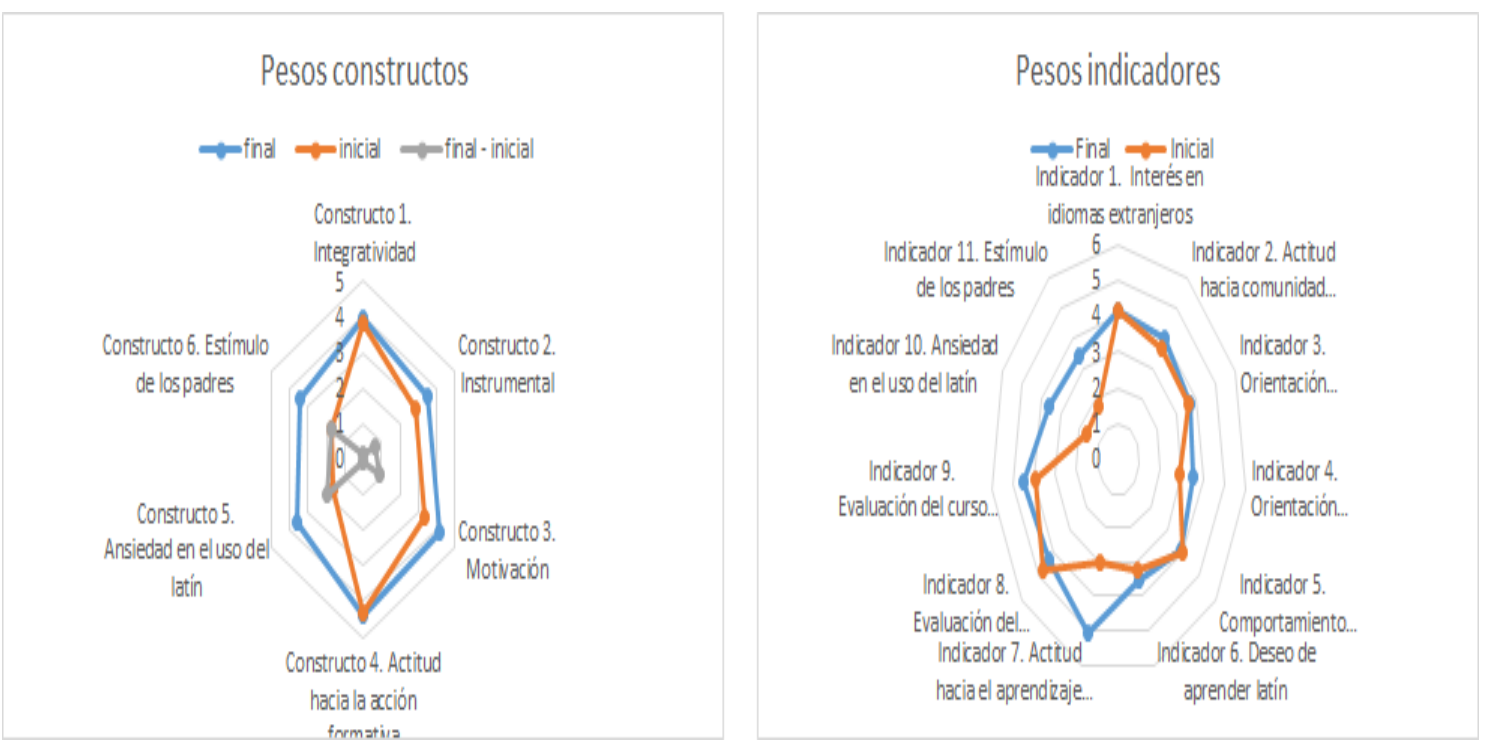

Figura 4. Comparativa de los pesos de los constructos e indicadores de la motivación-actitud al inicio y final de la acción formativa

\section{Discusión y Conclusiones}

En el presente estudio se ha puesto en práctica una nueva metodología didáctica de iniciación al latín que parece que mejora la motivación y la actitud positiva frente al aprendizaje del latín de los alumnos.

En relación con el objetivo general y objetivo específico cuatro, sobe la detección de un cambio significativo en la motivación a nivel global y a nivel de cada constructo e indicador, básicamente se observa que el grupo de alumnos al inicio de la acción formativa tenía una motivación-actitud media acerca de aprender latín como nueva lengua $(2,99 / 5)$. Después de la acción formativa, la motivación-actitud aumenta de forma significativa en casi en un punto, 0,88 (figura 1). Consideramos que el aumento es significativo cuando es de un punto, es decir, supera el doble de la desviación media y estándar que, en promedio es de medio punto (la varianza en promedio es $0,33)$. De forma más detallada:

1. Este aumento de la motivación-actitud parece que se debe fundamentalmente a un aumento significativo (de dos puntos sobre cinco) de la actitud positiva frente al aprendizaje del latín, indicador que forma parte de la motivación (constructo 4).

2. El estímulo de los padres es el segundo indicador que aumenta de forma significativa en 1,73 puntos. Curiosamente, al inicio de la acción formativa este indicador presentaba un valor bajo (1,7 puntos), mientras que al terminar la acción formativa el indicador se incrementa hasta un valor medio-alto $(3,6)$. Este incremento en el interés de los padres sólo puede explicarse porque los alumnos hayan compartido con sus padres la experiencia de aprendizaje de latín y, éstos, hayan percibido un cambio de actitud e interés en sus hijos. Es decir, parece que han sido los alumnos los que han cambiado la actitud de los padres frente a la nueva lengua.

3. El indicador 10 y constructo 5 , ansiedad en el uso del latín se incrementa en 2 puntos, pasando de tener un valor bajo, 1,6, a tener un valor medio-alto, 3,6. Este incremento es desconcertante y no se corresponde con el aumento también en dos puntos de la motivación y casi dos puntos del estímulo de los 
padres. Para poder corroborar que realmente hay un aumento de la ansiedad deberíamos haber incluido el resto de las afirmaciones del AMTB de este constructo. En todo caso, la afirmación textual que aparece en el cuestionario es "Me preocupa que me pregunten en clase de latín", es decir, lo que aumenta es la preocupación por no saber responder. Esto puede deberse a un nivel de exigencia grande por parte del profesor, puesto que disminuye ligeramente la valoración, muy alta, del profesor (indicador 8) al mismo tiempo que aumenta la valoración de la acción formativa (indicador 8). De forma alternativa podría explicarse por una mayor consciencia por parte del alumno de las dificultades que conlleva el aprendizaje de la nueva lengua en su aspecto morfológico (declinaciones) muy diferente al de su lengua materna.

4. El peso que tiene cada indicador y constructo en el valor global, al final de la acción formativa, de la motivación-actitud es, aproximadamente, el mismo. Es decir, todos contribuyen equitativamente a construir la motivación del alumno, aunque el peso de la motivación y actitud positiva acerca de la acción formativa son ligeramente mayores que el resto. Esto no era así, sin embargo, al comienzo de la acción formativa en la que parece que el peso del estímulo de los padres y la actitud hacia el aprendizaje del latín de los alumnos era menor que el resto de los indicadores.

Respecto a la evaluación de la validez de la encuesta, se observa que:

El indicador de orientación-motivación de integración hacia el aprendizaje de nuevas lenguas que razonablemente no debería cambiar, efectivamente, permanece estable.

Además, se ha comprobado que los valores de las afirmaciones en negativo son coherentes y complementarios con las afirmaciones correspondientes en positivo (pregunta 6: 2,1 puntos frente a 4,6 de su contrapartida; pregunta 7 : 1,9 puntos frente a 4,1; y, pregunta 15: 3 puntos frente a 4,9).

Finalmente, se observa una correspondencia entre la valoración cualitativa de la actitud de los alumnos realizada por el profesor en sus notas de campo (tabla 1, sección 4.3) y el incremento cuantitativo de la motivación en el indicador de actitud positiva frente al aprendizaje del latín. Como puede observarse en la tabla 1 , columna $4^{a}$, el grado de implicación del alumno aumenta claramente, al pasar de tener una actitud de atención e interés a una actitud de atención y participación en las actividades formativas.

En síntesis, de este estudio se puede concluir que la nueva metodología didáctica de iniciación al latín parece que mejora la motivación y, más concretamente, la actitud positiva frente al aprendizaje del latín de los alumnos. Posiblemente, como consecuencia de esto, también mejora el estímulo de los padres hacia su aprendizaje. En este sentido, parece que se ha logrado el objetivo didáctico del método que es hacer que el alumno entienda el funcionamiento del latín para que sienta que puede aprenderlo.

Asimismo, para los autores, ha resultado realmente útil utilizar el cuestionario AMTB porque no sólo les ha ayudado a sistematizar el procedimiento de evaluación de la eficacia del método respecto a la motivación sino también, les ha permitido comprender con más profundidad qué es lo que ha cambiado realmente. De esta forma, es posible diseñar futuras mejoras en los contenidos didácticos (lecciones) con el objetivo de mejorar otros aspectos de la motivación que resultan claves para 
iniciarse y continuar con el estudio del latín como es, concretamente, el deseo de aprender latín.

Finalmente, los autores esperan poder replicar este estudio con una muestra suficientemente grande para poder validar y analizar estadísticamente, de forma más fiable, la influencia de la metodología didáctica en la motivación.

\section{Referencias}

Gardner, RC. (1985). Social psychology and second language learning: The role of attitudes and motivation. London: Edward Arnold.

Gardner, RC. (2004) Attitude/Motivation Test Battery: International AMTB Research Project. London: University of Western Ontario.

Gardner, RC. (2006). "Motivation and Attitudes in Second Language Learning". In K. Brown, Encyclopaedia of Language \& Linguistics (pp.348-355). Oxford: Elsevier.

Gardner, RC., \& Lambert, WE. (1972). Attitudes and motivation: Second language learning. Newbury House.

Gardner, RC., \& Tremblay, PF. (1994). On motivation, research agendas, and theoretical frameworks. Modern Language Journal, 78, 359-368.

Márquez, M. (2015) Léxico de autores latinos en formato digital: elaboración de un modelo y su aplicación al léxico de los escritos consolatorios y elegías a la muerte del príncipe Juan, Tesis doctoral, Universidad Complutense de Madrid.

Márquez, M. y Chaves, B. (2016). A Latin Functionalist Dictionary as a Self-Learning Language Device: Previous Experiences to Digitalization. Educ. Sci. 6(3), 23.

Márquez, M. y Fernández-Pampillón, AM. (2016). El Diccionario funcionalista como instrumento de aprendizaje de una Lengua: experiencias previas a la digitalización, e-prints UCM.

Masgoret, AM., \& Gardner, RC. (2003). Attitudes, motivation, and second language learning: A meta-analysis of studies conducted by Gardner and Associates. Language Learning, 53(1), 123-163. 